\title{
Molecular Diagnostic Tests for Microsporidia
}

\author{
Kaya Ghosh ${ }^{1,2}$ and Louis M. Weiss ${ }^{1,3}$ \\ ${ }^{1}$ Department of Pathology, Albert Einstein College of Medicine, Bronx, NY 10461, USA \\ ${ }^{2}$ Department of Biological Sciences, Rutgers University, Newark, NY 07102, USA \\ ${ }^{3}$ Department of Medicine, Albert Einstein College of Medicine, Bronx, NY 10461, USA
}

Correspondence should be addressed to Louis M. Weiss, lmweiss@aecom.yu.edu

Received 31 March 2009; Accepted 12 May 2009

Recommended by Herbert B. Tanowitz

\begin{abstract}
The Microsporidia are a ubiquitous group of eukaryotic obligate intracellular parasites which were recognized over 100 years ago with the description of Nosema bombycis, a parasite of silkworms. It is now appreciated that these organisms are related to the Fungi. Microsporidia infect all major animal groups most often as gastrointestinal pathogens; however they have been reported from every tissue and organ, and their spores are common in environmental sources such as ditch water. Several different genera of these organisms infect humans, but the majority of infections are due to either Enterocytozoon bieneusi or Encephalitozoon species. These pathogens can be difficult to diagnose, but significant progress has been made in the last decade in the development of molecular diagnostic reagents for these organisms. This report reviews the molecular diagnostic tests that have been described for the identification of the microsporidia that infect humans.
\end{abstract}

Copyright (๑) 2009 K. Ghosh and L. M. Weiss. This is an open access article distributed under the Creative Commons Attribution License, which permits unrestricted use, distribution, and reproduction in any medium, provided the original work is properly cited.

\section{Introduction}

The Microsporidia are a phylum of over 1200 species representing at least 150 genera $[1,2]$. Since the mid1980s, these organisms have increasingly been implicated as agents of human disease, especially in their capacity as opportunistic pathogens in patients with HIV infection $[2,3]$ and other immunosuppressed individuals, such as those with organ transplantation or chemotherapy recipients [4]. To date, fourteen species in eight genera have been found to infect humans [5]. In HIV-positive patients, the most common clinical manifestation is chronic diarrhea and wasting due to enteric infection, but the spectrum of disease due to these pathogens is broad and includes hepatitis, peritonitis, keratoconjunctivits, sinusitis, bronchitis, pneumonia, cystitis, nephritis, myositis, encephalitis, and other cerebral infections [4]. In addition, microsporidia have also been reported to be etiologic in isolated case reports of urethritis, prostatic abscess, tongue ulcer, bone infection, and cutaneous infection [4]. There is an increasing appreciation that these organisms can also cause gastrointestinal and ocular infections in apparently immunocompetent individuals. Serosurveys $[6,7]$ suggest that microsporidiosis is common, but usually self-limiting or asymptomatic in the general population. While transmission routes have not been specifically documented in epidemiologic studies, there is evidence that infections can occur by multiple routes (enumerated in [2]) including waterborne, respiratory, sexual, congenital, zoonotic transmission, and in ocular infection by traumatic inoculation into the cornea.

All microsporidia produce an environmentally resistant spore which is capable of extruding its coiled, internal polar filament (i.e., polar tube) thereby inoculating its contents into a nearby host cell. Unique in structure and function, identification of the polar filament is diagnostic for the phylum. Due to the small size of the organisms, for example, several of the human-infecting species measure $1-2 \mu \mathrm{m}$ [4], diagnosis of microsporidiosis has traditionally relied on transmission electron microscopy (TEM) to identify the polar filament and other phylum- and species-specific ultrastructural characters. Although it remains the gold standard, TEM is labor-intensive and time-consuming, requiring expensive equipment, significant specialized expertise, and a dedicated histological staff working over the course of several days. It is also relatively insensitive, due to the small amount of tissue that can be examined and the lack of signal 
amplification. Light microscopy-based methods have also been developed and are faster and typically more sensitive than TEM, but they still require experienced pathologists for successful interpretation. These methods include routine histological stains such as the modified trichrome stain which is used alone or in combination with other stains such as Gram or Warthin-Starry silver [1]. Although these methods are more convenient than TEM for detecting microsporidia in body fluids and tissues, the internal polar filament is not easily identified using these techniques. Rather, diagnosis hinges mostly on the detection of the thick spore wall which is birefringent and provides selective staining characteristics with the modified trichrome stain. Chemofluorescent brighteners (e.g., Calcofluor White, Uvitex 2B, Fungifluor) have been used to target the chitin within the spore wall. While sensitive, the potential for cross reactivity with Fungi and artifactual material exists, especially in stool specimens. Thus, it has been recommended that chemofluorescent brighteners should be used in combination with traditional histological stains, to provide better sensitivity and specificity when examining stool specimens. However, even the best possible tissue preparation and staining for light microscopy rarely enables a microsporidian species-specific diagnosis. This is a critical shortcoming in light of the need for different treatments for the various microsporidian species that infect humans [8].

While TEM evidence of the polar filament or other ultrastructural features unique to the phylum is considered incontrovertible proof of microsporidiosis, a more specific diagnosis is not always possible on the basis of morphology alone. Especially in the case of closely related species, distinguishing characteristics may arise in only certain developmental stages of the organism, all of which may not be present in a particular clinical sample. While in vitro culture is conceivable as a tool to aid in diagnosis for several human-infecting species, culture methods are laborious, subject to contamination, and usually impractical; moreover, for Enterocytozoon bieneusi, the most common microsporidium found in humans, no in vitro culture system exists [9]. Thus, there exists a need for faster, more specific, and more accessible approaches to diagnosis in both clinical specimens and environmental samples.

Over the past decade or so, molecular biology-based procedures have been increasingly used in clinical settings for the diagnosis and characterization of microbial pathogens. These procedures are designed to detect either a nucleic acid sequence or antigen specific to the pathogen. Compared to traditional microscopy- or culture-based methods, molecular methods can offer the following potential advantages: increased sensitivity, by virtue of amplification of signal; greater specificity, when appropriate detection probes are employed; faster time-to-result; and greater ease of interpretation by nonspecialists [10]. While clinical laboratories still primarily rely on microscopy-based methods for the diagnosis of microsporidia, over the past fifteen years significant effort has been directed to the development of molecular methods in research laboratories. This article will review the progress toward molecular diagnostics of these emerging pathogens.

\section{Nucleic Acid-Based Detection Methods}

Nucleic acid-based detection methods utilize synthetic DNA molecules that are specific and complementary to a sequence in the DNA of the pathogen. The earliest methods employed labeled probes which hybridized to pathogen DNA and emitted a detectable (e.g., fluorescent) signal. Such DNA probe technologies are still in use today, although they have been largely supplanted by methods that amplify the target sequence; of these methods the most commonly utilized is the polymerase chain reaction (PCR) (for a historical perspective, see [11]). In PCR, the target pathogen DNA is bound by a specifically designed set of primers and copied over and over again in the presence of free nucleotides by a themostable polymerase enzyme. The amplification of the target pathogen DNA (i.e., amplicon) confers two advantages: improved detection sensitivity relative to probebased methods and facilitation of downstream analyses (e.g., restriction analysis, sequencing) of the amplicon.

Techniques for sample preparation for the molecular diagnosis of microsporidia have been reviewed in detail in Weiss and Vossbrinck [12]. The technique used to extract DNA for amplification can significantly affect the sensitivity of a PCR diagnostic technique. Nucleic acids may be extracted from clinical samples such as tissue biopsies, corneal scrapings, duodenal aspirations, and urine specimens as well as in vitro cultures with commercial DNA extraction kits (e.g., those manufactured by Qiagen, Santa Clara, Calif, Usa, or Promega, Madison, Wis, Usa) or by routine procedures such as proteinase $\mathrm{K}$ digestion followed by phenol-chloroform extraction and ethanol precipitation [13]. DNA may also be isolated from paraffin-embedded material by standard methods [14] or with commercial kits such as DexPAT (Takera Biochemical, Berkeley Calif, Usa). DNA has been successfully amplified from modified trichrome-stained [15] and decades-old Giemsa-stained [16] microscope slides by scraping the material off of the slides followed by mechanical disruption of the microsporidia (using glass beads) and subsequent DNA extraction using standard techniques.

The isolation and amplification of DNA from stool samples is more challenging, generally requiring mechanical disruption and/or harsh extraction conditions. Successful reported methods include subjection to $0.5 \%$ sodium hypochlorite [17], chitinase [17], lyticase [18], guanidine thiocyanate [19-21], 10\% formalin or M potassium hydroxide [17, 22, 23], dithiothreitol [22], hexadecyltrimethylammoniuim bromide [24], or boiling the samples (Ombrouck et al. [25] . Stool samples frequently contain inhibitors of polymerase enzymes [26]. If they are not removed by the above methods, dilution of the samples (Ombrouck et al.,[27]) or guanidine thiocyanate treatment [19] may be warranted. An extraction-free template preparation method for stool samples has also been developed [28] using FTA filters impregnated with denaturants, chelating agents, and free-radical traps, which apparently causes most cells to lyse on contact and enables debris and other inhibitory factors to be washed away from the DNA trapped on the filter. In one study this FTA filter method allowed the detection of 
800 microsporidian spores per milliliter of stool by a PCR technique (Subrungruang et al., [29]) .

In order to apply PCR-based diagnostics to a pathogen, some genetic sequence information must be known in advance. The human-infecting microsporidia are a diverse group of "emerging" pathogens, and the available genetic information on these organisms is limited but everincreasing. For the majority of the microsporidia GenBank sequence data on their rRNA genes is the only genetic information available. The number of microsporidian genes deposited in GenBank has grown from less than 200 in 1999 (surveyed in [12]) to almost 6000 today. Approximately twothousand of these are the genome sequence-related predicted genes proposed by Katinka et al. [30] in their landmark genomic sequencing of the human pathogen Encephalitzoon cuniculi, the first and to-date only microsporidium genome to be completely sequenced. For the closely related species Encephalitozoon hellem, there are 75 entries. Recently, a genomic survey of the essentially noncultivatable pathogen Enterocytozoon bieneusi resulted in the addition of another three-thousand hypothetical genes, some of which are homologs to the genes identified in Enc. cuniculi [31]. For the other eleven human-infecting species of microsporidia, between zero and a few dozen genes have been deposited in GenBank.

Due to the availability of sequence information as well as the presence of conserved and variable regions within the rRNA genes, PCR-based methods have typically utilized primers to this gene for the characterization of the microsporidia. The first such report of the use of conserved rRNA primers was of that of the cloning of the small subunit (SSU) rDNA of Vairimorpha necatrix, a pathogen of agricultural pests [32]. Primers complementary to conserved sequences within this gene were used to amplify and subsequently obtain sequence data on the rRNA gene of several human-infecting microsporidia, including Enc. cuniculi, Enc. hellem, Enc. intestinalis, Ent. bieneusi, and Vittaforma corneae (reviewed in $[33,34]$ ). These rRNA genes have been reported by Katinka et al. [30] to be present in more than twenty copies in the Enc. cuniculi genome, and therefore, provide an increase in sensitivity (over single copy genes) for use in diagnostic PCR tests.

Diagnostic studies using primers to the various rRNA genes of microsporidia have been reviewed by Weiss and Vossbrinck [12] and Franzen and Muller [35]. The sequences of many of the primer pairs used for the amplification of various microsporidia, along with the recommended annealing temperatures for PCR and the expected amplicon size, are compiled in Table 1 (adapted from [12]). Some of these primers are species-specific whereas others are more general primer sets that amplify all of the Encephalitozoonidae. For some of these primer sets downstream restriction analysis, wherein the amplicon is digested into smaller pieces by specific restriction enzymes, is required for species specific diagnoses.

PCR has also been useful for the identification of the previously unknown microsporidia in human and veterinary infections. Using phylogenetically conserved primers amplifying the small subunit (SSU), large subunit
(LSU), and intergenic spacer (IGS) regions, it has been possible to clone and then sequence portions of the rRNA gene of uncharacterized microsporidia from biopsy specimens (Table 2, adapted from [12]). These rRNA sequence data can then be used for phylogenetic analysis using BLAST and similar in silico programs comparing the unknown sequence to the rRNA sequences on various microsporidia available in GenBank. The primers in Table 2 form the basis of a "molecular toolbox" which allows the cloning of rRNA genes from novel species or strains of microsporidia. The primer pairs V1(18f)::1492r and 530f::580r are considered "universal" in that they are usually successful in amplifying unknown rRNA genes for novel species or strains of microsporidia ([36], also see [12]).

Several investigators have published diagnostic procedures for microsporidia which use real-time PCR [33, 34, 53, 54, 56]. Real-time PCR, which detects accumulating amplicons in real time via interacting either fluorescent dyes or fluorescence-labelled probes, has the advantage of being quantitative over a broad dynamic range. In addition, it typically employs a multiwell format and dispenses with postamplification processing of the sample, which increases throughput and reduces the risk of contamination inherent in PCR [57]. Hester et al. [53] used a probe specific for the small subunit rRNA of the genus Encephalitozoon and species-specific primers for Enc. cuniculi, Enc. hellem, and Enc. intestinalis. While their method was validated only for purified microsporidian DNA, it could be adapted for clinical samples. Another study utilized pan-Encephalitozoon primers specific to small subunit rRNA and a guanidine thiocyanate-based extraction system designed for an automated workstation to detect Enc. cuniculi, Enc. hellem, and Enc. intestinalis from stool specimens [56]. The assay was sensitive (detection limit between $10^{2}$ to $10^{3}$ spores $/ \mathrm{mL}$ ) and reflective of infection intensity (linear range was between $10^{3}$ and $10^{7}$ spores $/ \mathrm{mL}$ ). In addition, melting curve analyses of the amplicons readily allowed differentiation of the three Enc. species., which is useful for multiple or unknown infections. In 2003, a real-time PCR assay using primers for Enc. intestinalis small subunit rRNA was used to detect this pathogen from known clinical samples, including stools, urine, tissue biopsies, bronchopulmonary specimens, and blood [33, 34]. Using control reference spores, the detection limit was estimated to be 20 spores per milliliter, which was sufficient to detect a relatively low-intensity blood infection suggesting that an infection was disseminated [33, 34]. Finally, a multiplex realtime PCR assay has also been reported to simultaneously detect Ent. bieneusi, Enc. cuniculi, Enc. hellem, and Enc. intestinalis from both fresh and formalin-fixed stool with primers for the intergenic region and small subunit rRNA of Ent. bieneusi and Encephalitozoon species., respectively [54]. Ent. bieneusi was detected in 30 of 33 known microsporidiapositive samples. The study included a range of negative and positive controls to verify the assay specificity and guard against false negatives due to inhibitors potentially present in stool or to the presence of extraneous DNA, respectively.

A few studies have also utilized fluorescent in situ hybridization (FISH-) based methods to detect microsporidia. 


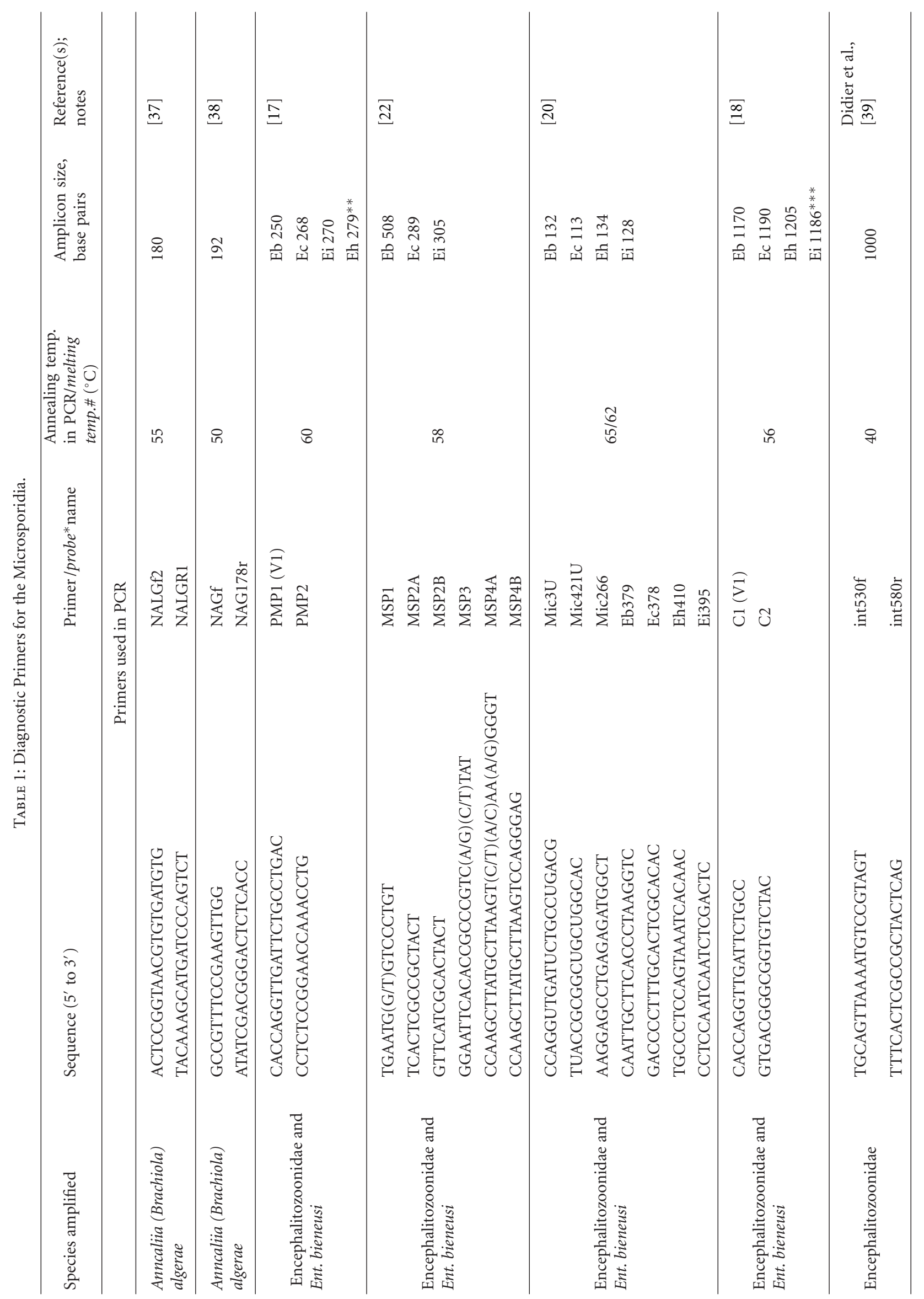




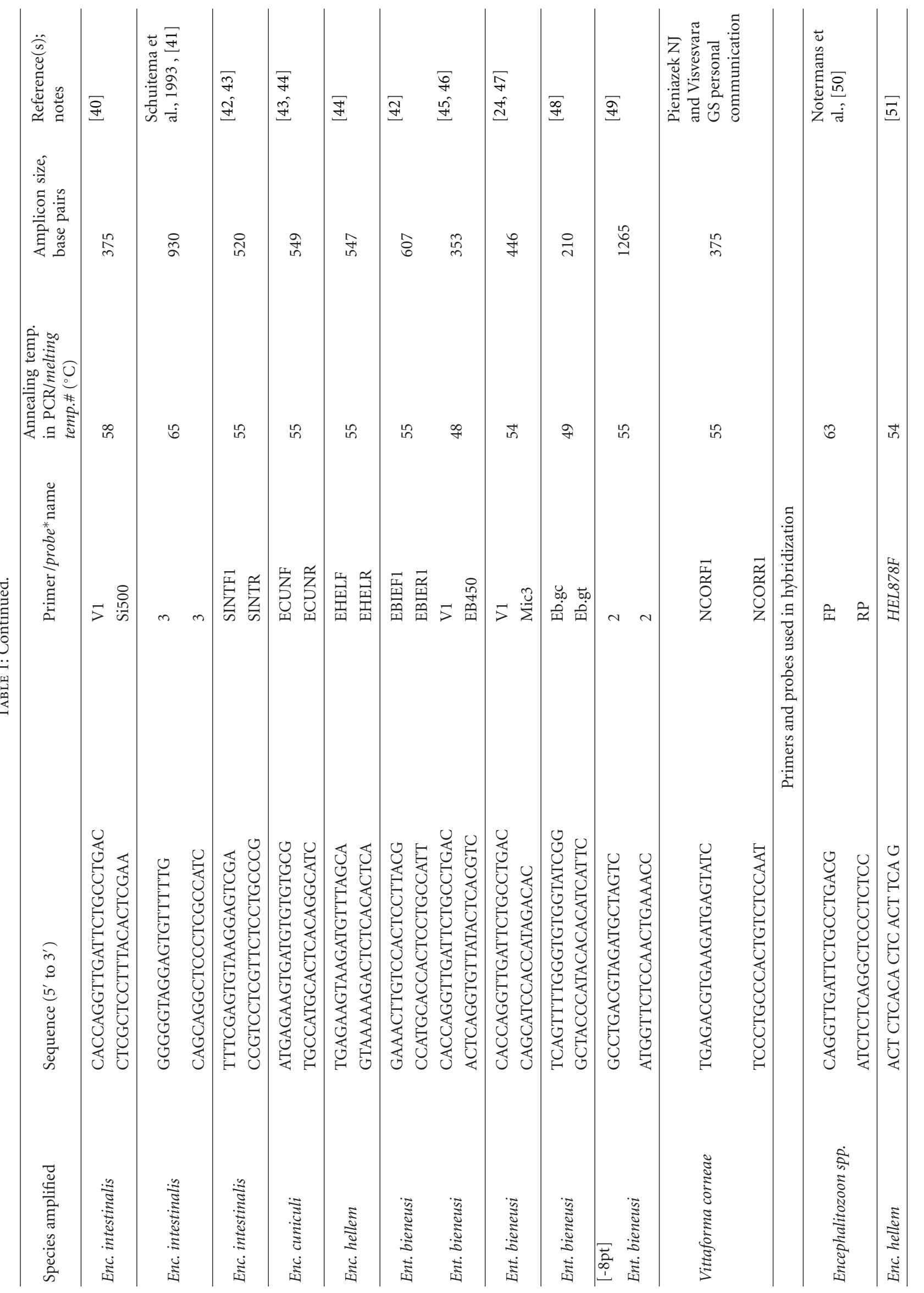




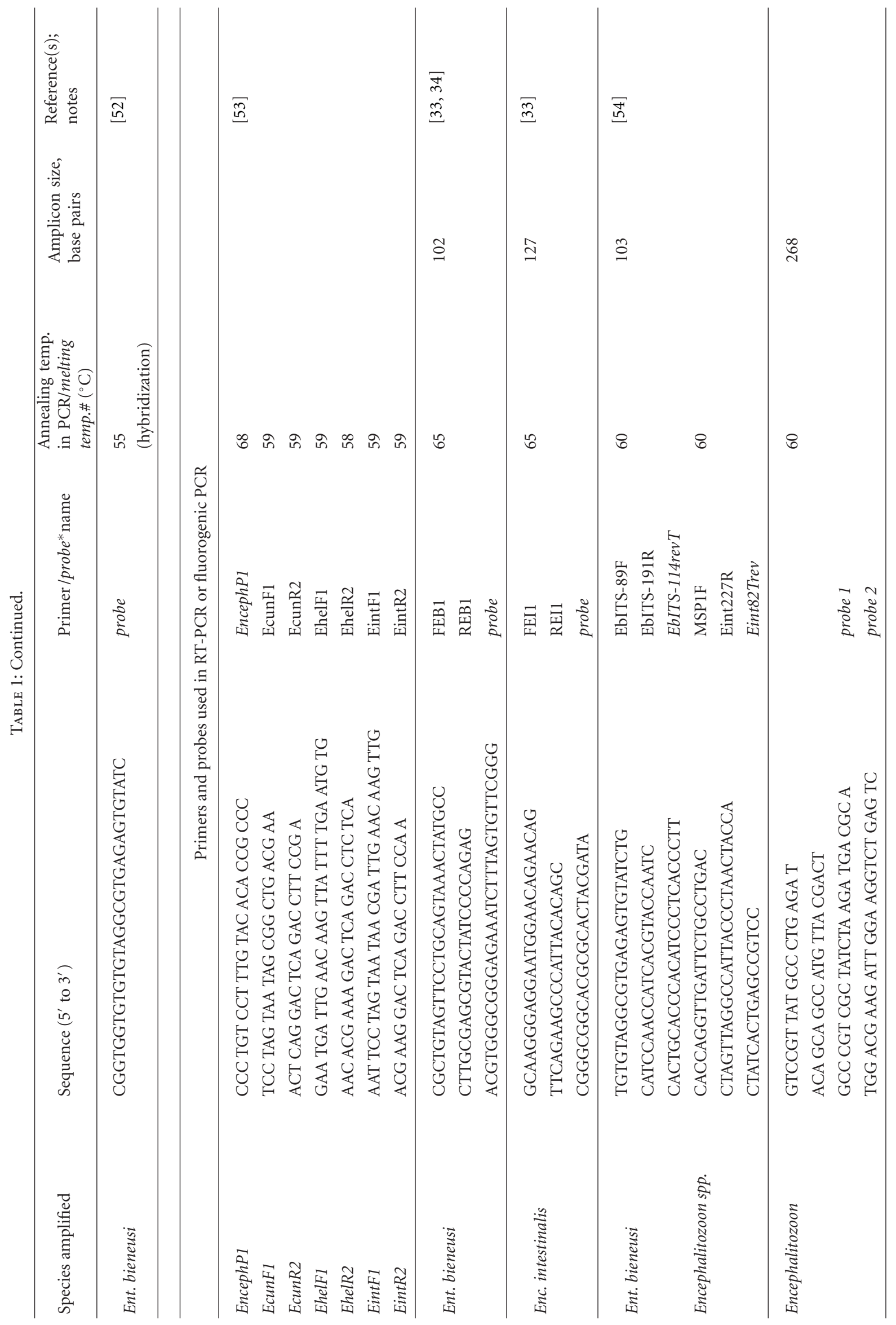




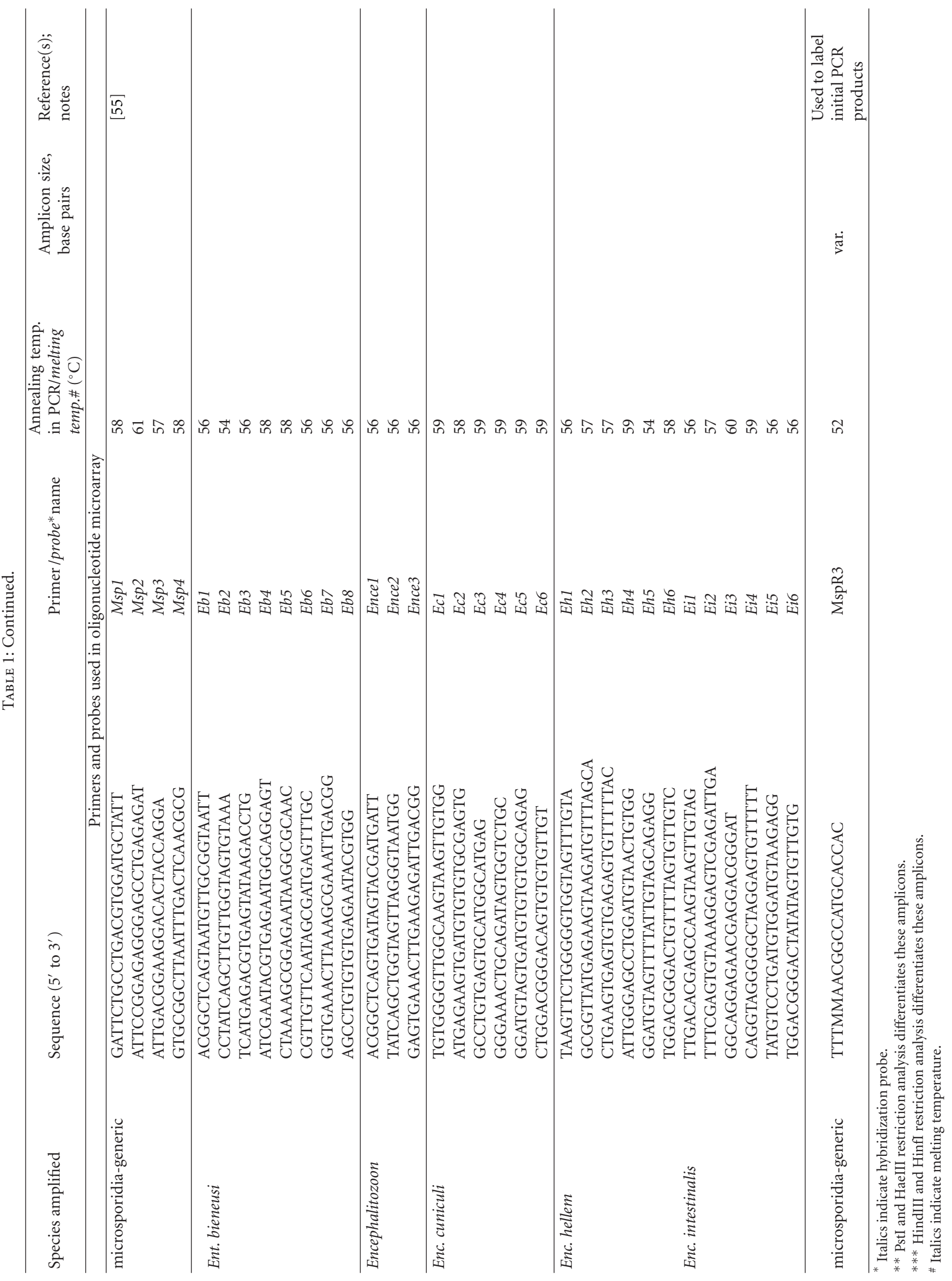


TABLE 2: Primers for the identification and sequencing of microsporidian rRNA ${ }^{1}$ Genes.

\begin{tabular}{lc}
\hline ss $^{2} 18 \mathrm{f}^{3}$ & CACCAGGTTGATTCTGCC \\
ss18sf & GTTGATTCTGCCTGACGT \\
ss350f & CCAAGGA(T/C)GGCAGCAGGCGCGAAA \\
ss350r & TTTCGCGCCTGCTGCC(G/A)TCCTTG \\
ss530f & GTGCCAGC(C/A)GCCGCGG \\
ss530r & CCGCGG(T/G)GCTGGCAC \\
ss1047r & AACGGCCATGCACCAC \\
ss1061f & GGTGGTGCATGGCCG \\
ss1492r & GGTTACCTTGTTACGACTT (Universal primer) \\
ss1537 & TTATGATCCTGCTAATGGTTC \\
ls212r1 & GTT(G/A)GTTTCTTTTCCTC \\
ls212r2 & AATCC(G/A/T/C)(G/A)GTT(G/A)GTTTCTTTTCCTC \\
ls580r & GGTCCGTGTTTCAAGACGG \\
\hline
\end{tabular}

1- Primers $18 \mathrm{f}$ and $1492 \mathrm{r}$ amplify most of the small subunit rRNA of the microsporidia. Primes $530 \mathrm{f}$ and $212 \mathrm{r} 1$ or $212 \mathrm{r} 2$ are used to amplify the small subunit rRNA and the ITS region. The remaining primers are used to sequence, with overlap, the forward and reverse strands of the entire small subunit rRNA and ITS region. 1s580r amplifies a variable region of the $5^{\prime}$ end of the large subunit rRNA gene of many microsporidia (e.g., Nosema and Vairimorpha) but it does not work on all microsporidia. ss1537 allows sequencing closer to the $3^{\prime}$ end of the small subunit rRNA of many but not all microsporidia. ss $350 \mathrm{f}$ and ss350r may not be needed for sequencing reactions if $18 \mathrm{f}$ and $530 \mathrm{r}$ provide sufficient overlap to obtain clear sequence data.

2- ss: denotes primers in the small subunit rRNA gene,

ls: denotes primers in the large subunit rRNA gene,

f: forward primer (positive strand),

r: reverse primer (negative strand).

3- Similar to V1 primer.

Adapted from [12].

Essentially, FISH technology utilizes a fluorescence-labeled probe that binds to complementary nucleic acid (DNA or RNA) in the specimen [10]. In contrast to PCR, general morphological and spatial information regarding probe-binding in the specimen may be retained because the procedure is performed in situ. FISH has been used with probes against the small subunit or intergenic regions of microsporidia rRNA to detect Ent. bieneusi and Enc. hellem [24, 51, 52]. These methods were used successfully with archived formalin-fixed, paraffin-embedded (FFPE) clinical samples and detected either more microsporidia-positive samples or more infected cells within samples than traditional histochemical staining. In the case of Ent. bieneusi, characteristic staining of parasites in a supranuclear location within jejunal biopsy epithelial cells [24] and staining of developmental forms [52] contributed to the certainty of diagnosis. Although FISH is an attractive procedure due to the multifaceted information, it can provide two factors seriously hamper its potential for general use in clinical diagnostic settings. Firstly, it is rather laborious and technically challenging, requiring deparaffinization, dehydration, and rehydration (of FFPE samples), digestion by proteinases to make the nucleic acid accessible to the probe, labeling and overnight hybridization of the probe, blocking, counterstaining, and many wash steps prior to viewing with an epifluorescenceequipped microscope. Secondly, it is less sensitive than PCR by orders of magnitude due to the lack of amplification of original signal (i.e., the nucleic acid target). Nonetheless, it may prove particularly useful for environmental samples wherein discrimination of live versus dead organisms is important, which can be afforded by designing probes for the less-stable RNA rather than DNA (discussed in [51]).

Of interest is the report of the development of an oligonucleotide microarray to simultaneously detect Ent. bieneusi, Enc. cuniculi, Enc. hellem, and Enc. intestinalis from clinical samples [55]. Such microarrays were originally developed for genomewide expression analysis but have recently been applied to molecular diagnostics $[10,58]$. Microarray technology commonly employs an array of target-complementary oligonucleotides printed on a "chip" to which fluorescence-labelled nucleic acid from the sample is hybridized; the degree of fluorescence correlates to the abundance of the sample DNA. Because of the array format and the analog nature of fluorescence intensity, this technology is intrinsically high-throughput and somewhat quantitative, respectively. Wang et al. [55] capitalized on these advantages and combined them with the sensitivity of PCR by first using conserved, family-specific primers to amplify 1.3-kb microsporidia rRNA fragments from unextracted, FTA-filtered clinical fecal samples before hybridization to a microsporidia microarray. Multiple specific probes were then used to confer genus- and species-level hybridization profiles to the assay and to increase sensitivity by decreasing amplicon size (i.e., in a "nested" fashion). The array was able to simultaneously detect all four species of microsporidia at a sensitivity of $10^{2}$ spores per $100 \mu \mathrm{L}$ of fecal sample. In a survey of 20 fecal samples from AIDS patients suffering from diarrhea of unknown etiology, 12 samples were microsporidia-positive, and all but one were apparently multiply-infected. No masking effect by the more abundant species was evident, and the probe hybridization profile for each species offers a tentative assessment of infection intensity. The printing of four individual microarrays per slide increases the potential throughput of this technique.

\section{Antigen-Based Detection Methods}

Antigen-based detection methods such as the immunofluorescence assays (IFAs), ELISA, and immunoblot use antibodies from experimentally immunized animals to recognize characteristic pathogen specific antigens. IFA can be used in situ on fixed specimens but needs to be examined using fluorescence microscopy. Immunoblot or ELISA tests examine an homogenate of the specimen. Antibodies may be either polyclonal (i.e., purified from animal sera and directed against various epitopes of the protein, and possibly containing other, nonspecific antibodies which can increase background signal) or monoclonal (purified from cell culture supernatants).

A number of monoclonal and polyclonal antibodies against human-infecting microsporidia including Ent. bieneusi, Enc. cuniculi, Enc. hellem, and Enc. intestinalis have been developed [59-65]. Most often these antibodies have been directed against the spore wall or polar tube of the 
Microsporidia. Some of these antibodies have demonstrated cross-reactivity among various species of microsporidia by IFA or immunoblot. While some investigators have reported IFA tests that had an equal sensitivity to a reference PCR (e.g., [64]), the majority of investigators believe that IFA tests are less sensitive than PCR based methods. In any case, the specificity and sensitivity depend to a great extent on the antibody itself and the care with which the various steps (e.g. fixation, blocking, and washes) are executed. Antibodybased detection is best used as a supplement to conventional histological techniques, and in difficult cases nucleotidebased detection should be utilized as well.

\section{Antibody-Based Detection Methods}

Serologic tests such as the enzyme-linked immunosorbent assay (ELISA), immunoblot, and agglutination-based tests $[66,67]$ which can detect circulating antibody are not currently recommended for diagnostic purposes due to variable expression of antibodies in immunocompromised patients, the inability to discriminate between acute and past infections [68], the high prevalence of anti-microsporidian antibodies in apparently healthy, immunocompetent populations $[6,7]$, and cross-reactivity of antibodies between different species. However, these serologic analyses may be useful to diagnose subclinical infections in prospective transplant donors or patients who may be at risk for reactivation of infection due to impending immune compromise (discussed in [8]).

\section{Detection of Microsporidia from Environ- mental Samples}

Because many species of microsporidia are enteric pathogens in humans and animals and are transmitted as environmentally resistant spores [69], it is likely that waterborne transmission of these parasites occurs. Humanpathogenic microsporidia have been detected in surface water, groundwater, and tertiary agricultural effluent [23, 70-75], which poses a contamination risk to drinking, recreational, and agricultural water supplies. Indeed, in 1999 there was a confirmed waterborne outbreak of microsporidiosis that affected both immune-compromised and immunecompetent individuals [76]. As a result of such studies, the U.S. Environmental Protection Agency included the microsporidia on its two most recent Candidate Contaminant Lists (CCL-1 and -2) in 1998 and 2005, respectively. The CCL-2 currently consists of eight other candidate microbiological agents and 42 chemical agents which are known or anticipated to be present in public water systems and which may require regulation under the Safe Drinking Water Act.

Waterborne protozoa are usually detected from largevolume water samples by filter-based or centrifugal concentration followed by purification and molecular or microscopic identification of the organism from the concentrated material [77]. Currently, methods for the enrichment of microsporidia in water samples have not been standardized, but relatively expedient concentration of spores has been achieved by continuous flow centrifugation (CFC) [78] or continuous separation channel centrifugation [79]. In the case of water samples, purification of spores by immunomagnetic separation (IMS), which utilizes pathogen-specific antibody-coated beads prior to detection by real-time PCR, was shown to be $78 \%-90 \%$ sensitive for seeded spores in ultrapure water [78], although the paucity of commercially available anti-microsporidia antibodies currently limits the accessibility of this approach. In all cases requiring detection of microsporidia from turbid samples such as feces-impacted or otherwise turbid environmental water, the small size of human-pathogenic microsporidia impedes detection sensitivity, as it necessitates a reduction in filter pore size which increases membrane-fouling, thus effectively decreasing the volume of water that can be filtered [78]. In the case of feces or heavily feces-impacted wastewater, a similar problem arises in that although smaller, filtered, or unfiltered volumes may be analyzed for the sake of convenience, and to minimize the effect of PCR inhibitors usually present therein [26], such small volumes may not be representative of the entire sample or may be inadequate for detection of low-intensity contaminations (discussed in [80]). Nonetheless, detection limits of $10^{2}$ to $10^{3}$ spores per milliliter of feces or wastewater were achieved by sucrose-flotation purification followed by DNA extraction using commercial kits and PCR [80], a significant improvement over previously reported methods even for less turbid samples [71, 81].

While waterborne microsporidia likely pose the greater environmental threat, nonaquatic dispersal of microsporidia is also a public health concern. Spores have been identified on fresh produce in Poland such as berries and other fruits, sprouts, and green-leaf vegetables [82], perhaps as a consequence of microsporidial contamination of agricultural irrigation waters [74] or sewage-sludge end products used as fertilizer $[83,84]$. Three species of Encephalitozoonidae were detected by FISH at levels likely to be infective for humans. In urban settings in Europe and North America, human-infecting microsporidia have been identified from pigeon fecal droppings [83-86]. The genotype of one isolate was found to match that of a previously reported humaninfecting isolate [86], and in one study, 11\% of pigeon fecal samples were found to be Ent. bieneusi-positive [85]. Graczyk et al. $[83,84]$ estimated that a person could inhale $10^{3}$ viable, aerosolized spores in 30 minutes of occupational or incidental exposure to heavily pigeon excrement-contaminated surfaces. In addition, Mathis et al. [87] demonstrated Ent. bieneusi in feces of farm dogs and cats; diagnostic PCR suggested that the strains are closely related to human isolates. These findings support the notion that human microsporidiosis is a zoonotic disease $[69,88,89]$ and highlight the utility of molecular methods to identify new sources of risk to human health.

\section{Conclusion}

The potential of molecular diagnostics and particularly nucleic acid-based diagnostics to exceed traditional methods 
in terms of sensitivity, specificity, speed, and reproducibility has already achieved proof-of-concept for other pathogens $[10,11]$. Indeed, this has been demonstrated for the microsporidia in molecular detection studies that processed corresponding specimens for light microscopy (e.g., [24, 51]). Although a blinded, multicenter evaluation of detection methods for the microsporidia conducted in 1998 (Rinder et al. [90]) revealed only a modest sensitivity advantage of PCR (89\%) over light microscopy $(80 \%)$, the greatest differences were seen between individual laboratories. Thus it is likely that as the molecular diagnostic methods are perfected over time and clinical diagnostic technicians become accustomed to them, their advantages will become more apparent. While the costs of such technology and requisite training of staff may seem initially prohibitive, the embedded costs of delayed and nonspecific or incorrect diagnosis to both patients and health care systems should be considered (see [11]).

In summary, molecular detection methods for the microsporidia described herein are potentially more sensitive, specific, and depend less on the subjectivity of the observer than traditional microscopy-based methods. Additionally, sophisticated nucleotide-based methods such as real-time PCR and oligonucleotide microarrays are intrinsically higher-throughput and quantitative, enabling simultaneous analysis of specimens for multiple pathogens as well as a tentative assessment of infection intensity. Although the time-to-result and reproducibility in clinical diagnostic settings have yet to be evaluated, a modest learning curve should be expected considering the "emerging" nature of these pathogens (see [91]). Looking ahead as more physiological insight into these pathogens is afforded by recent genomic sequencing projects $[30,31]$, these technologies may even be adapted as necessary to applications such as strain-genotyping and drug sensitivity-profiling.

\section{References}

[1] L. S. Garcia, "Laboratory identification of the microsporidia," Journal of Clinical Microbiology, vol. 40, no. 6, pp. 1892-1901, 2002.

[2] L. M. Weiss, "Microsporidia: emerging pathogenic protists," Acta Tropica, vol. 78, no. 2, pp. 89-102, 2001.

[3] A. Cali, "General microsporidian features and recent findings on AIDS isolates," Journal of Protozoology, vol. 38, no. 6, pp. 625-630, 1991.

[4] C. Franzen and A. Müller, "Microsporidiosis: human diseases and diagnosis," Microbes and Infection, vol. 3, no. 5, pp. 389400, 2001.

[5] E. S. Didier, "Microsporidiosis: an emerging and opportunistic infection in humans and animals," Acta Tropica, vol. 94, no. 1, pp. 61-76, 2005.

[6] N. R. Bergquist, G. Stintzing, L. Smedman, T. Waller, and T. Andersson, "Diagnosis of encephalitozoonosis in man by serological tests," British Medical Journal, vol. 288, no. 6421, p. 902, 1984

[7] T. van Gool, J. C. M. Vetter, B. Weinmayr, A. Van Dam, F. Derouin, and J. Dankert, "High seroprevalence of Encephalitozoon species in immunocompetent subjects," Journal of Infectious Diseases, vol. 175, no. 4, pp. 1020-1024, 1997.
[8] E. S. Didier and L. M. Weiss, "Microsporidiosis: current status," Current Opinion in Infectious Diseases, vol. 19, no. 5, pp. 485-492, 2006.

[9] G. S. Visvesvara, "In vitro cultivation of microsporidia of clinical importance," Clinical Microbiology Reviews, vol. 15, no. 3, pp. 401-413, 2002.

[10] G. W. Procop, "Molecular diagnostics for the detection and characterization of microbial pathogens," Clinical Infectious Diseases, vol. 45, supplement 2, pp. S99-S111, 2007.

[11] S. Yang and R. E. Rothman, "PCR-based diagnostics for infectious diseases: uses, limitations, and future applications in acute-care settings," Lancet Infectious Diseases, vol. 4, no. 6, pp. 337-348, 2004.

[12] L. M. Weiss and C. R. Vossbrinck, "Molecular biology, molecular phylogeny, and molecular diagnostic approaches to the microsporidia," in The Microsporidia and Microsporidiosis, M. Wittner and L. M. Weiss, Eds., pp. 129-171, American Society for Microbiology, Washington, DC, USA, 1999.

[13] F. M. Ausubel, R. Brent, R. E. Kingston, et al., Current Protocols in Molecular Biology, John Wiley \& Sons, New York, NY, USA, 1997.

[14] M. A. Innis, D. H. Gelfand, J. J. Sninsky, and T. J. White, PCR Protocols: A Guide to Methods and Applications, Academic Press, San Diego, Calif, USA, 1990.

[15] K. S. Chan and T. H. Koh, "Extraction of microsporidial DNA from modified trichrome-stained clinical slides and subsequent species identification using PCR sequencing," Parasitology, vol. 135, no. 6, pp. 701-703, 2008.

[16] M. Hylis, J. Weiser, M. Obornik, and J. Vavra, "DNA isolation from museum and type collection slides of microsporidia," Journal of Invertebrate Pathology, vol. 88, no. 3, pp. 257-260, 2005.

[17] D. P. Fedorko and Y. M. Hijazi, "Application of molecular techniques to the diagnosis of microsporidial infection," Emerging Infectious Diseases, vol. 2, no. 3, pp. 183-191, 1996.

[18] L. Raynaud, F. Delbac, V. Broussolle, et al., "Identification of Encephalitozoon intestinalis in travelers with chronic diarrhea by specific PCR amplification," Journal of Clinical Microbiology, vol. 36, no. 1, pp. 37-40, 1998.

[19] R. Boom, C. J. A. Sol, M. M. M. Salimans, C. L. Jansen, P. M. E. Wertheim-van Dillen, and J. van der Noordaa, "Rapid and simple method for purification of nucleic acids," Journal of Clinical Microbiology, vol. 28, no. 3, pp. 495-503, 1990.

[20] N. P. Kock, H. Petersen, T. Fenner, et al., "Species-specific identification of microsporidia in stool and intestinal biopsy specimens by the polymerase chain reaction," European Journal of Clinical Microbiology and Infectious Diseases, vol. 16, no. 5, pp. 369-376, 1997.

[21] A. H. Talal, D. P. Kotler, J. M. Orenstein, and L. M. Weiss, "Detection of Enterocytozoon bieneusi in fecal specimens by polymerase chain reaction analysis with primers to the smallsubunit rRNA," Clinical Infectious Diseases, vol. 26, no. 3, pp. 673-675, 1998.

[22] S. Katzwinkel-Wladarsch, M. Lieb, W. Heise, T. Loscher, and H. Rinder, "Direct amplification and species determination of microsporidian DNA from stool specimens," Tropical Medicine and International Health, vol. 1, no. 3, pp. 373-378, 1996.

[23] J. M. Sparfel, C. Sarfati, O. Liguory, et al., "Detection of microsporidia and identification of Enterocytozoon bieneusi in surface water by filtration followed by specific PCR," Journal of Eukaryotic Microbiology, vol. 44, no. 6, p. 78S, 1997.

[24] A. Carville, K. Mansfield, G. Widmer, et al., "Development and application of genetic probes for detection of Enterocytozoon 
bieneusi in formalin-fixed stools and in intestinal biopsy specimens from infected patients," Clinical and Diagnostic Laboratory Immunology, vol. 4, no. 4, pp. 405-408, 1997.

[25] C . Ombrouck, L. Ciceron, and I. Desportes-Livag, "Specific and rapid detection of Microsporidia in stool specimens from AlDS patients by PCR," Parasite, vol. 3, no. 1, pp. 85-6, 1996.

[26] L. Monteiro, D. Bonnemaison, A. Vekris, et al., "Complex polysaccharides as PCR inhibitors in feces: helicobacter pylori model," Journal of Clinical Microbiology, vol. 35, no. 4, pp. 995998, 1997.

[27] C . Ombrouck, L. Ciceron, S. Biligui, et al., "Specific PCR assay for direct detection of intestinal microsporidia Enterocytozoon bieneusi and Encephalitozoon intestinalis in fecal specimens from human immunodeficiency virusinfected patient," J Clin Microbiol., vol. 35, no. 3, pp. 652-5, 1997.

[28] P. A. Orlandi and K. A. Lampel, "Extraction-free, filterbased template preparation for rapid and sensitive PCR detection of pathogenic parasitic protozoa," Journal of Clinical Microbiology, vol. 38, no. 6, pp. 2271-2277, 2000.

[29] I. Subrungruan, M. Mungthi, P. Chavalitshewinkoon-Petmit, R. Rangsi, T. Naaglor, and S. Leelayoova, "Evaluation of DNA extraction and PCR methods for detection of Enterocytozoon bienuesi in stool specimens," J Clin Microbiol., vol. 42, no. 8, pp. 3490-4, 2004.

[30] M. D. Katinka, S. Duprat, E. Cornillott, et al., "Genome sequence and gene compaction of the eukaryote parasite Encephalitozoon cuniculi," Nature, vol. 414, no. 6862, pp. 450-453, 2001.

[31] D. E. Akiyoshi, H. G. Morrison, S. Lei, et al., "Genomic survey of the non-cultivatable opportunistic human pathogen, Enterocytozoon bieneusi," PLoS Pathogens, vol. 5, no. 1, article e1000261, 2009.

[32] C. R. Vossbrinck, J. V. Maddox, S. Friedman, B. A. DebrunnerVossbrinck, and C. R. Woese, "Ribosomal RNA sequence suggests microsporidia are extremely ancient eukaryotes," Nature, vol. 326, no. 6111, pp. 411-414, 1987.

[33] J. Menotti, B. Cassinat, R. Porcher, C. Sarfati, F. Derouin, and J.-M. Molina, "Development of a real-time polymerase-chainreaction assay for quantitative detection of Enterocytozoon bieneusi DNA in stool specimens from immunocompromised patients with intestinal microsporidiosis," Journal of Infectious Diseases, vol. 187, no. 9, pp. 1469-1474, 2003.

[34] J. Menotti, B. Cassinat, C. Sarfati, O. Liguory, F. Derouin, and J.-M. Molina, "Development of a real-time PCR assay for quantitative detection of Encephalitozoon intestinalis DNA," Journal of Clinical Microbiology, vol. 41, no. 4, pp. 1410-1413, 2003.

[35] C. Franzen and A. Müller, "Molecular techniques for detection, species differentiation, and phylogenetic analysis of microsporidia," Clinical Microbiology Reviews, vol. 12, no. 2, pp. 243-285, 1999.

[36] C. R. Vossbrinck and B. A. Debrunner-Vossbrinck, "Molecular phylogeny of the Microsporidia: ecological, ultrastructural and taxonomic considerations," Folia Parasitologica, vol. 52, no. 1-2, pp. 131-142, 2005.

[37] G. S. Visvesvara, M. Belloso, H. Moura, et al., "Isolation of Nosema algerae from the cornea of an immunocompetent patient," Journal of Eukaryotic Microbiology, vol. 46, no. 5, p. 10S, 1999.

[38] A. Cali, P. M. Takvorian, S. Lewin, et al., "Brachiola vesicularum, n. g., n. sp., a new microsporidium associated with AIDS and myositis," Journal of Eukaryotic Microbiology, vol. 45, no. 3, pp. 240-251, 1998.
[39] ES. Didier, LB. Roger, AD. Brush, S. Wong, V. Traina-Dorge, and D. Bertucc, "Diagnosis of disseminated microsporidian Encephalitozoon hetlem infection bv PCR-Southern analvsis and successful treatment with albendazole and fumaqillin," $J$ Clin Microbiol., vol. 34, no. 4, pp. 947-52, 1996.

[40] L. M. Weiss, X. Zhu, A. Cali, H. B. Tanowitz, and M. Wittner, "Utility of microsporidian rRNA in diagnosis and phylogeny: a review," Folia Parasitologica, vol. 41, no. 2, pp. 81-90, 1994.

[41] A. R. J. Schuitema, R. A. Hartskeerl, T. van Gool, R. Laxminarayan, and W. J. Terpstra, "Application of the polymerase chain reaction for the diagnosis of microsporidiosis," AIDS, vol. 7, supplement 3, pp. S57-S61, 1993.

[42] A. J. da Silva, S. B. Slemenda, G. S. Visvesvara, et al., "Detection of septata intestinalis (microsporidia) cali et al. 1993 using polymerase chain reaction primers targeting the small subunit ribosomal RNA coding region," Molecular Diagnosis, vol. 2, no. 1, pp. 47-52, 1997.

[43] M. A. De Groote, G. Visvesvara, M. L. Wilson, et al., "Polymerase chain reaction and culture confirmation of disseminated Encephalitozoon cuniculi in a patient with AIDS: successful therapy with albendazole," Journal of Infectious Diseases, vol. 171, no. 5, pp. 1375-1378, 1995.

[44] G. S. Visvesvara, G. J. Leitch, A. J. da Silva, et al., "Polyclonal and monoclonal antibody and PCR-amplified small-subunit rRNA identification of a microsporidian, Encephalitozoon hellem, isolated from an AIDS patient with disseminated infection," Journal of Clinical Microbiology, vol. 32, no. 11, pp. 2760-2768, 1994.

[45] X. Zhu, M. Wittner, H. B. Tanowitz, D. Kotler, A. Cali, and L. M. Weiss, "Small subunit rRNA sequence of Enterocytozoon bieneusi and its potential diagnostic role with use of the polymerase chain reaction," Journal of Infectious Diseases, vol. 168, no. 6, pp. 1570-1575, 1993.

[46] C. M. Coyle, M. Wittner, D. P. Kotler, et al., "Prevalence of microsporidiosis due to Enterocytozoon bieneusi and Encephalitozoon (Septata) intestinalis among patients with AIDS-related diarrhea: determination by polymerase chain reaction to the microsporidian small-subunit rRNA gene," Clinical Infectious Diseases, vol. 23, no. 5, pp. 1002-1006, 1996.

[47] K. G. Mansfield, A. Carville, D. Shvetz, J. MacKey, S. Tzipori, and A. A. Lackner, "Identification of an Enterocytozoon bieneusi-like microsporidian parasite in simianimmunodeficiency-virus-inoculated macaques with hepatobiliary disease," American Journal of Pathology, vol. 150, no. 4, pp. 1395-1405, 1997.

[48] J. N. Velasquez, S. Carnevale, E. A. Guarnera, et al., "Detection of the microsporidian parasite Enterocytozoon bieneusi in specimens from patients with AIDS by PCR," Journal of Clinical Microbiology, vol. 34, no. 12, pp. 3230-3232, 1996.

[49] F. David, A. R. J. Schuitema, C. Sarfati, et al., "Detection and species identification of intestinal microsporidia by polymerase chain reaction in duodenal biopsies from human immunodeficiency virus-infected patients," Journal of Infectious Diseases, vol. 174, no. 4, pp. 874-877, 1996.

[50] DW. Notermans, R. Peek, MD. de Jong, EM. WentinkBonnema, R. Boom, and T. van Gool, "Detection and identification of Enterocytozoon bieneusi and Encephalitozoon species in stool and urine specimens by PCR and differential hybridization.," J Clin Microbiol, vol. 43, no. 2, pp. 610-4, 2005.

[51] J. D. Hester, H. D. A. Lindquist, A. M. Bobst, and F. W. Schaefer III, "Fluorescent in situ detection of Encephalitozoon 
hellem spores with a 6-carboxyfluorescein-labeled ribosomal RNA-targeted oligonucleotide probe," Journal of Eukaryotic Microbiology, vol. 47, no. 3, pp. 299-308, 2000.

[52] J. N. Velásquez, S. Carnevale, J. H. Labbé, A. Chertcoff, M. G. Cabrera, and W. Oelemann, "In situ hybridization: a molecular approach for the diagnosis of the microsporidian parasite Enterocytozoon bieneusi," Human Pathology, vol. 30, no. 1, pp. 54-58, 1999.

[53] J. D. Hester, M. Varma, A. M. Bobst, M. W. Ware, H. D. A. Lindquist, and F. W. Schaefer III, "Species-specific detection of three human-pathogenic microsporidial species from the genus Encephalitozoon via fluorogenic $5^{\prime}$ nuclease PCR assays," Molecular and Cellular Probes, vol. 16, no. 6, pp. 435-444, 2002.

[54] J. J. Verweij, R. ten Hove, E. A. T. Brienen, and L. van Lieshout, "Multiplex detection of Enterocytozoon bieneusi and Encephalitozoon spp. in fecal samples using real-time PCR," Diagnostic Microbiology and Infectious Disease, vol. 57, no. 2, pp. 163-167, 2007.

[55] Z. Wang, P. A. Orlandi, and D. A. Stenger, "Simultaneous detection of four human pathogenic microsporidian species from clinical samples by oligonucleotide microarray," Journal of Clinical Microbiology, vol. 43, no. 8, pp. 4121-4128, 2005.

[56] D. M. Wolk, S. K. Schneider, N. L. Wengenack, L. M. Sloan, and J. E. Rosenblatt, "Real-time PCR method for detection of Encephalitozoon intestinalis from stool specimens," Journal of Clinical Microbiology, vol. 40, no. 11, pp. 3922-3928, 2002.

[57] P. T. Monis and S. Giglio, "Nucleic acid amplificationbased techniques for pathogen detection and identification," Infection, Genetics and Evolution, vol. 6, no. 1, pp. 2-12, 2006.

[58] A. Loy and L. Bodrossy, "Highly parallel microbial diagnostics using oligonucleotide microarrays," Clinica Chimica Acta, vol. 363, no. 1-2, pp. 106-119, 2006.

[59] H. D. Luján, J. T. Conrad, C. G. Clark, et al., "Detection of microsporidia spore-specific antigens by monoclonal antibodies," Hybridoma, vol. 17, no. 3, pp. 237-243, 1998.

[60] L. Mo and M. Drancourt, "Monoclonal antibodies for specific detection of Encephalitozoon cuniculi," Clinical and Diagnostic Laboratory Immunology, vol. 11, no. 6, pp. 1060-1063, 2004.

[61] K. Furuya, S. Miwa, M. Omura, et al., "Mouse monoclonal immunoglobulin $\mathrm{E}$ antibodies specific for the microsporidian Encephalitozoon cuniculi polar tube protein 1," Hybridoma, vol. 27, no. 3, pp. 153-157, 2008.

[62] G. P. Croppo, G. S. Visvesvara, G. J. Leitch, S. Wallace, and D. A. Schwartz, "Identification of the microsporidian Encephalitozoon hellem using immunoglobulin G monoclonal antibodies," Archives of Pathology and Laboratory Medicine, vol. 122, no. 2, pp. 182-186, 1998.

[63] Q. Zhang, I. Singh, A. Sheoran, et al., "Production and characterization of monoclonal antibodies against Enterocytozoon bieneusi purified from rhesus macaques," Infection and Immunity, vol. 73, no. 8, pp. 5166-5172, 2005.

[64] I. Singh, A. S. Sheoran, Q. Zhang, A. Carville, and S. Tzipori, "Sensitivity and specificity of a monoclonal antibodybased fluorescence assay for detecting Enterocytozoon bieneusi spores in feces of simian immunodeficiency virus-infected macaques," Clinical and Diagnostic Laboratory Immunology, vol. 12, no. 10, pp. 1141-1144, 2005.

[65] A. S. Sheoran, X. Feng, I. Singh, et al., "Monoclonal antibodies against Enterocytozoon bieneusi of human origin," Clinical and Diagnostic Laboratory Immunology, vol. 12, no. 9, pp. 11091113, 2005.
[66] C. N. Jordan, A. M. Zajac, K. S. Snowden, and D. S. Lindsay, "Direct agglutination test for Encephalitozoon cuniculi," Veterinary Parasitology, vol. 135, no. 3-4, pp. 235-240, 2006.

[67] I. F. Abou El Naga, M. R. Gaafar, L. A. El-Zawawy, D. El-Said, and S. F. Mossallam, "The utility of direct agglutination (DAT) and fast agglutination screening (FAST) tests in serodiagnosis of experimental microsporidiosis," Journal of the Egyptian Society of Parasitology, vol. 38, no. 3, pp. 903-918, 2008.

[68] Z. Kucerova-Pospisilova and O. Ditrich, "The serological surveillance of several groups of patients using antigens of Encephalitozoon hellem and E. cuniculi antibodies to microsporidia in patients," Folia Parasitologica, vol. 45, no. 2, pp. 108-112, 1998.

[69] M. Wittner and L. M. Weiss, The Microsporidia and Microsporidiosis, American Society for Microbiology, Washington, DC, USA, 1999.

[70] S. W. Avery and A. H. Undeen, "The isolation of microsporidia and other pathogens from concentrated ditch water," Journal of the American Mosquito Control Association, vol. 3, no. 1, pp. 54-58, 1987.

[71] S. E. Dowd, C. P. Gerba, and I. L. Pepper, "Confirmation of the human-pathogenic microsporidia Enterocytozoon bieneusi, Encephalitozoon intestinalis, and Vittaforma corneae in water," Applied and Environmental Microbiology, vol. 64, no. 9, pp. 3332-3335, 1998.

[72] S. E. Dowd, D. John, J. Eliopolus, et al., "Confirmed detection of Cyclospora cayetanesis, Encephalitozoon intestinalis and Cryptosporidium parvum in water used for drinking," Journal of Water Health, vol. 1, no. 3, pp. 117-123, 2003.

[73] S. Fournier, O. Liguory, M. Santillana-Hayat, et al., "Detection of microsporidia in surface water: a one-year follow-up study," FEMS Immunology and Medical Microbiology, vol. 29, no. 2, pp. 95-100, 2000.

[74] J. A. Thurston-Enriquez, P. Watt, S. E. Dowd, R. Enriquez, I. L. Pepper, and C. P. Gerba, "Detection of protozoan parasites and microsporidia in irrigation waters used for crop production," Journal of Food Protection, vol. 65, no. 2, pp. 378-382, 2002.

[75] S. Coupe, K. Delabre, R. Pouillot, S. Houdart, M. SantillanaHayat, and F. Derouin, "Detection of Cryptosporidium, Giardia and Enterocytozoon bieneusi in surface water, including recreational areas: a one-year prospective study," FEMS Immunology and Medical Microbiology, vol. 47, no. 3, pp. 351359, 2006.

[76] L. Cotte, M. Rabodonirina, F. Chapuis, et al., "Waterborne outbreak of intestinal microsporidiosis in persons with and without human immunodeficiency virus infection," Journal of Infectious Diseases, vol. 180, no. 6, pp. 2003-2008, 1999.

[77] D. S. Zarlenga and J. M. Trout, "Concentrating, purifying and detecting waterborne parasites," Veterinary Parasitology, vol. 126, no. 1-2, pp. 195-217, 2004.

[78] R. M. Hoffman, D. M. Wolk, S. K. Spencer, and M. A. Borchardt, "Development of a method for the detection of waterborne microsporidia," Journal of Microbiological Methods, vol. 70, no. 2, pp. 312-318, 2007.

[79] M. A. Borchardt and S. K. Spencer, "Concentration of Cryptosporidium, microsporidia and other water-borne pathogens by continuous separation channel centrifugation," Journal of Applied Microbiology, vol. 92, no. 4, pp. 649-656, 2002.

[80] A. M. Kahler and J. A. Thurston-Enriquez, "Human pathogenic microsporidia detection in agricultural samples: method development and assessment," Parasitology Research, vol. 100, no. 3, pp. 529-538, 2007. 
[81] X. Li, K. W. Tate, L. A. Dunbar, B. Huang, and E. R. Atwill, "Efficiency for recovering Encephalitozoon intestinalis spores from waters by centrifugation and immunofluorescence microscopy," Journal of Eukaryotic Microbiology, vol. 50, supplement, pp. 579-580, 2003.

[82] S. Jedrzejewski, T. K. Graczyk, A. Slodkowicz-Kowalska, L. Tamang, and A. C. Majewska, "Quantitative assessment of contamination of fresh food produce of various retail types by human-virulent microsporidian spores," Applied and Environmental Microbiology, vol. 73, no. 12, pp. 4071-4073, 2007.

[83] T. K. Graczyk, F. E. Lucy, L. Tamang, and A. Miraflor, "Human enteropathogen load in activated sewage sludge and corresponding sewage sludge end products," Applied and Environmental Microbiology, vol. 73, no. 6, pp. 2013-2015, 2007.

[84] T. K. Graczyk, D. Sunderland, A. M. Rule, et al., "Urban feral pigeons (Columba livia) as a source for air- and waterborne contamination with Enterocytozoon bieneusi spores," Applied and Environmental Microbiology, vol. 73, no. 13, pp. 43574358, 2007.

[85] A. Bart, E. M. Wentink-Bonnema, E. R. Heddema, J. Buijs, and T. van Gool, "Frequent occurrence of human-associated microsporidia in fecal droppings of urban pigeons in Amsterdam, The Netherlands," Applied and Environmental Microbiology, vol. 74, no. 22, pp. 7056-7058, 2008.

[86] M. Haro, F. Izquierdo, N. Henriques-Gil, et al., "First detection and genotyping of human-associated microsporidia in pigeons from urban parks," Applied and Environmental Microbiology, vol. 71, no. 6, pp. 3153-3157, 2005.

[87] A. Mathis, A. C. Breitenmoser, and P. Deplazes, "Detection of new Enterocytozoon genotypes in faecal samples of farm dogs and a cat," Parasite, vol. 6, no. 2, pp. 189-193, 1999.

[88] E. S. Didier, M. E. Stovall, L. C. Green, P. J. Brindley, K. Sestak, and P. J. Didier, "Epidemiology of microsporidiosis: sources and modes of transmission," Veterinary Parasitology, vol. 126, no. 1-2, pp. 145-166, 2004.

[89] A. Curry, "Human microsporidial infection and possible animal sources," Current Opinion in Infectious Diseases, vol. 12, no. 5, pp. 473-480, 1999.

[90] H. Rinder, K. Janitschke, H. Aspöock, et al., "Blinded, externally controlled multicenter evaluation of liaht microscoclv and PCR for detection of microsporidiain stool specimens. The Diaunostic Multicenter Study Group on Microsporidia.," J Clin Microbiol, vol. 36, no. 6, pp. 1814-8, 1998.

[91] J. Dong, J. P. Olano, J. W. McBride, and D. H. Walker, "Emerging pathogens: challenges and successes of molecular diagnostics," Journal of Molecular Diagnostics, vol. 10, no. 3, pp. 185-197, 2008. 


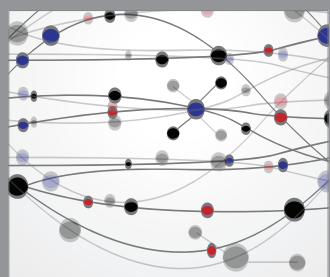

The Scientific World Journal
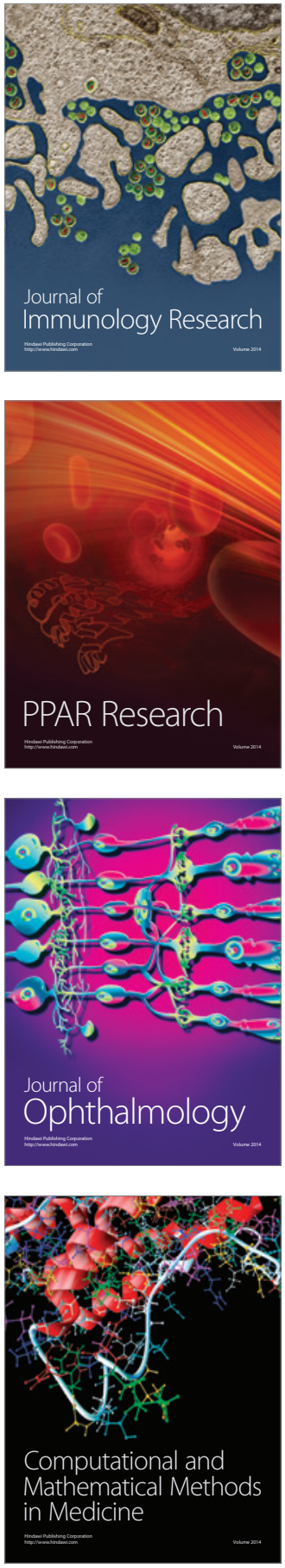

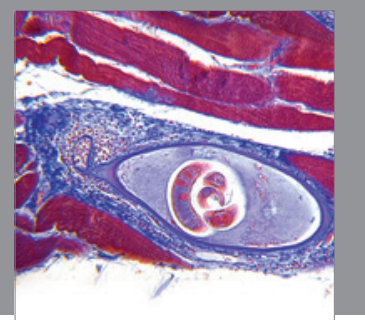

Gastroenterology

Research and Practice
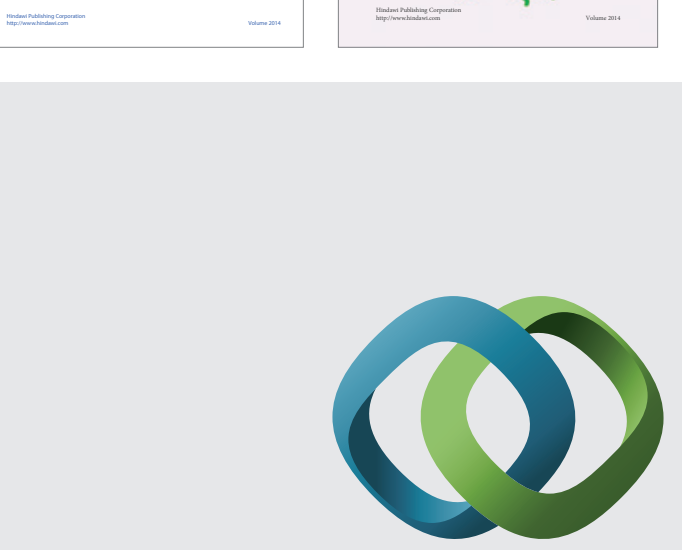

\section{Hindawi}

Submit your manuscripts at

http://www.hindawi.com
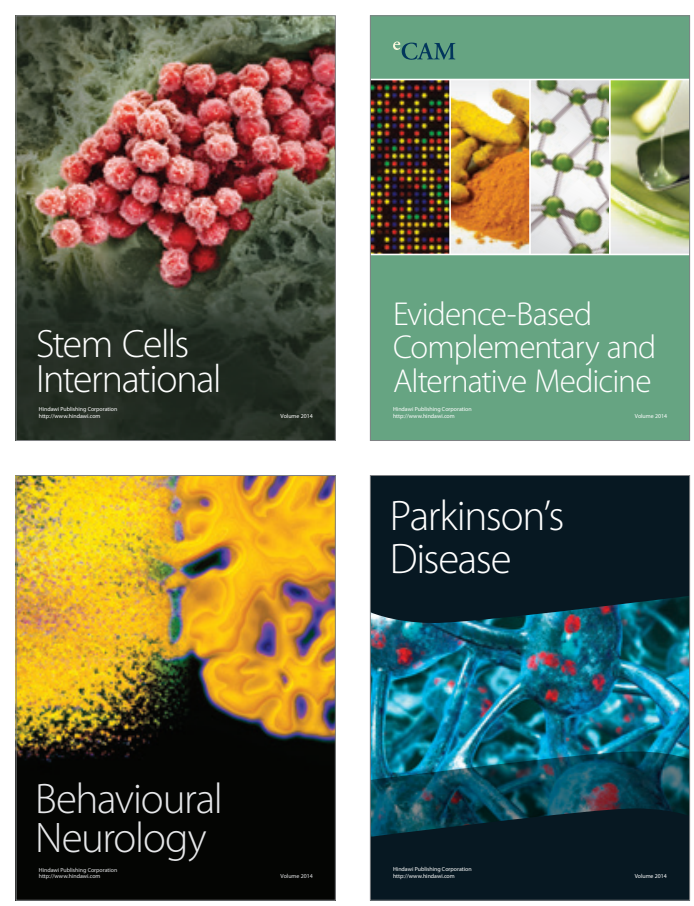

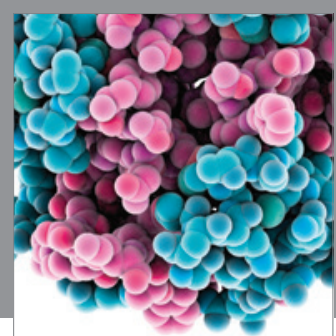

Journal of
Diabetes Research

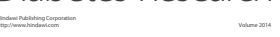

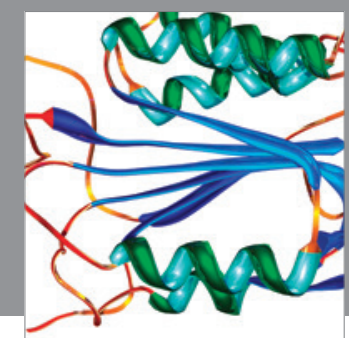

Disease Markers
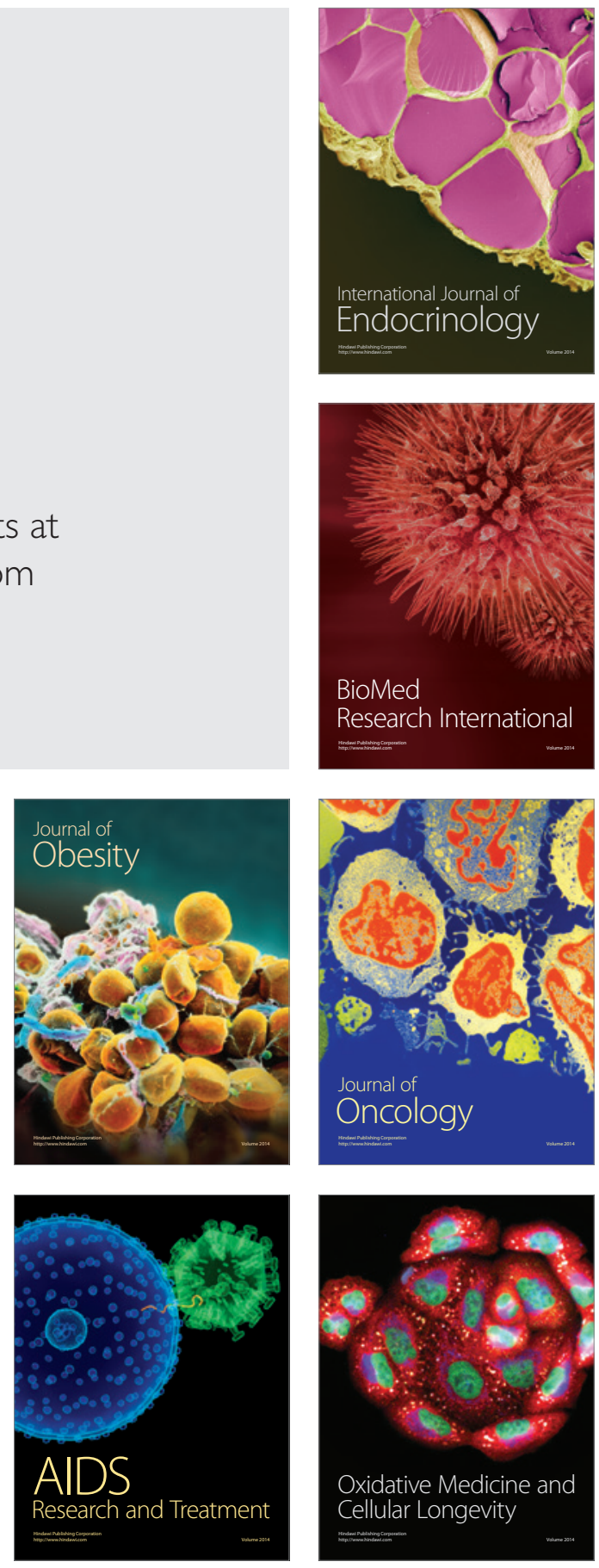